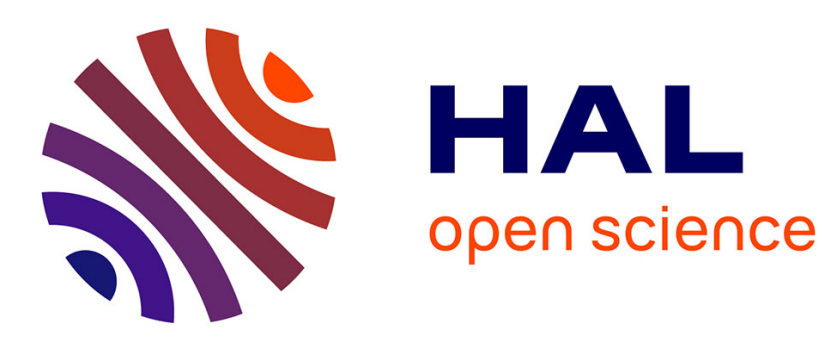

\title{
The liH2+ quasimolecule. a comparison between the configuration interaction and the OEDM approaches
}

L.F. Errea, L. Méndez, A. Riera, M. Yáñez, J. Hanssen, C. Harel, A. Salin

\section{To cite this version:}

L.F. Errea, L. Méndez, A. Riera, M. Yáñez, J. Hanssen, et al.. The liH2+ quasimolecule. a comparison between the configuration interaction and the OEDM approaches. Journal de Physique, 1985, 46 (5), pp.709-718. 10.1051/jphys:01985004605070900 . jpa-00210012

\section{HAL Id: jpa-00210012 https://hal.science/jpa-00210012}

Submitted on 1 Jan 1985

HAL is a multi-disciplinary open access archive for the deposit and dissemination of scientific research documents, whether they are published or not. The documents may come from teaching and research institutions in France or abroad, or from public or private research centers.
L'archive ouverte pluridisciplinaire HAL, est destinée au dépôt et à la diffusion de documents scientifiques de niveau recherche, publiés ou non, émanant des établissements d'enseignement et de recherche français ou étrangers, des laboratoires publics ou privés. 


\title{
The $\mathrm{LiH}^{2+}$ quasimolecule. A comparison between the configuration interaction and the OEDM approaches
}

\author{
L. F. Errea, L. Méndez, A. Riera, M. Yáñez \\ Departamento de Química Física y Química Cuántica, Universidad Autónoma de Madrid, 28049 Madrid, Spain \\ J. Hanssen, C. Harel and A. Salin \\ Laboratoire des Collisions Atomiques (*), Université de Bordeaux I, 33405 Talence, France
}

(Reçu le 9 juillet 1984, révisé le 14 décembre, accepté le 4 janvier 1985)

\begin{abstract}
Résumé. - Nous présentons dans cet article une comparaison directe entre la méthode OEDM (One-Electron Diatomic Molecule) et une approche moléculaire classique, basée sur la méthode d'interaction de configuration (CI) utilisant une base construite à partir de produits anti-symétrisés d'orbitales gaussiennes (GTO), pour le traitement d'une collision atomique à deux électrons. La quasi-molécule $\mathrm{LiH}^{2+}$ a été choisie comme exemple. Nous étudions les avantages de l'emploi d'un hamiltonien écranté pour définir les orbitales OEDM. Le diagramme de corrélation énergétique pour les sous-systèmes singulet et triplet est discuté ainsi que le caractère des états moléculaires intervenant dans la collision $\mathrm{Li}^{2+}(1 \mathrm{~s})+\mathrm{H}(1 \mathrm{~s})$. Nous montrons que la comparaison entre les deux méthodes permet d'interpréter le comportement des couplages obtenus dans la méthode d'interaction de configuration et d'attribuer sans équivoque les symboles moléculaires au diagramme de corrélation. Bien que l'image intuitive "d'un électron actif " soit utile pour l'étude du système collisionnel considéré, l'interaction entre les orbitales internes et externes joue un rôle non négligeable pour déterminer tant les couplages que le diagramme d'énergie.
\end{abstract}

\begin{abstract}
We present a direct comparison of the One-Electron Diatomic Molecule (OEDM) method with the conventional molecular approach, based on a full-configuration-interaction (CI) method using a basis set made up of symmetry adapted anti-symmetrized products of Gaussian type orbitals (GTO), for the treatment of atomic collisions involving two-electrons. The quasimolecule $\mathrm{LiH}^{2+}$ has been chosen as an example. We have studied the advantage of a screened Hamiltonian to define the OEDM orbitals. The energy correlation diagrams for the singlet and triplet subsystems are discussed, and also the physical character of the molecular states involved in the $\mathrm{Li}^{2+}(1 \mathrm{~s})+\mathrm{H}(1 \mathrm{~s})$ collision. We show that the comparison between both methods permits to interpret the detailed behaviour of the couplings obtained in the full configuration interaction method and to assign unambiguously molecular symbols in the correlation diagrams. Even though the intuitive " one active electron " picture is useful for the present collision system, the inner-outer orbital interaction is shown to be significant for both the energy diagram and couplings.
\end{abstract}

\section{Introduction.}

In previous work $[1,2]$ we have shown that antisymmetrized products of One-Electron Diatomic Orbitals (OEDM) are very convenient for the expansion of the wave function that describes the evolution of the electronic state in an ion-atom collision. This « OEDM

(*) Equipe de Recherche C.N.R.S. no 260. expansion " has been successfully applied to treat $\mathrm{He}_{2}^{2+}, \mathrm{HeH}^{+}$and $\mathrm{OHe}^{8+}$ quasimolecular systems. The OEDM orbitals corresponding to an electronic state in the field of two Coulomb charges, one may think $a$ priori that the method would be more appropriate when transitions between Rydberg-type molecular orbitals are responsible for the collision dynamics [3] - or equivalently, using a quantum chemistry language, when correlation effects are unimportant in those dynamics. Still the results obtained for the 
collisions $\mathrm{He}^{2+}+\mathrm{He}\left(1 \mathrm{~s}^{2}\right)$ and $\mathrm{H}^{+}+\mathrm{He}\left(1 \mathrm{~s}^{2}\right)$ show that the validity of the method is not restricted to such cases. Before going into the task of generalizing the method for an $N$ electron problem, we propose in the present paper a more thorough evaluation of the method for the two electron system $\mathrm{Li}^{2+}-\mathrm{H}$. We have therefore carried out a direct comparison between expansions onto OEDM and symmetry adapted products of Gaussian Type Orbitals (GTO).

Obviously the type of expansion used to define configurations for a Configuration Interaction method may seem at first sight more a technical than a basic problem. For example we could have used as well Slater type orbitals or elliptic orbitals in the present study. Furthermore, the OEDM orbitals can be considered as a particular type of contracted elliptical orbitals (as for atoms, hydrogenic orbitals are contracted Slater type orbitals). However the OEDM expansions have some specific features which make them quite useful both from a practical point of view and for a better understanding of the intuitive physics of an ion-atom collision and we summarize these briefly.

The qualitative description of atomic collisions has often been based on correlation diagrams build along the rules put forward by Lichten [14] and Barat and Lichten [15]. Even if these rules must be modified or extended in some cases (e.g. [20]), they are still a very good starting point to define the set of states that one has to take into account for a quantitative evaluation. As these correlation diagrams are based on OEDM correlation rules, their realization in terms of an OEDM expansion for actual calculations is particularly simple. In fact, the quantitative usefulness of OEDM expansions is at the root of the adequacy of qualitative correlation diagrams such as are often employed in conventional CI expansions. This fact in itself makes a comparison between the OEDM and full CI expansions with GTO, STO, etc. interesting. Furthermore we also show in the present work that the detailed structure of the radial and rotational coupling matrix elements in the GTO-CI expansion can be understood by using their relation with those evaluated in the OEDM expansion whereas this can usually be done only in a qualitative manner by inspection of the expansion coefficients [5-8].

The $\mathrm{LiH}^{2+}$ molecule has been chosen as an example. This system is well suited to an application of the OEDM method : in the states involved in the $\mathrm{Li}^{2+}$. $\mathrm{H}(1 \mathrm{~s})$ collision, the distinction can be made between an inner and outer orbital(Fig. 1). Hence the advantage of employing a screened Hamiltonian to define the OEDM orbitals can be explored. Furthermore, we avoid the complications due to autoionization that appear for systems with larger charges (see e.g. $\mathrm{O}^{8+}-\mathrm{He}$ in [3]).

The study of the collision process itself will be the subject of a further paper.

Atomic units are used throughout.
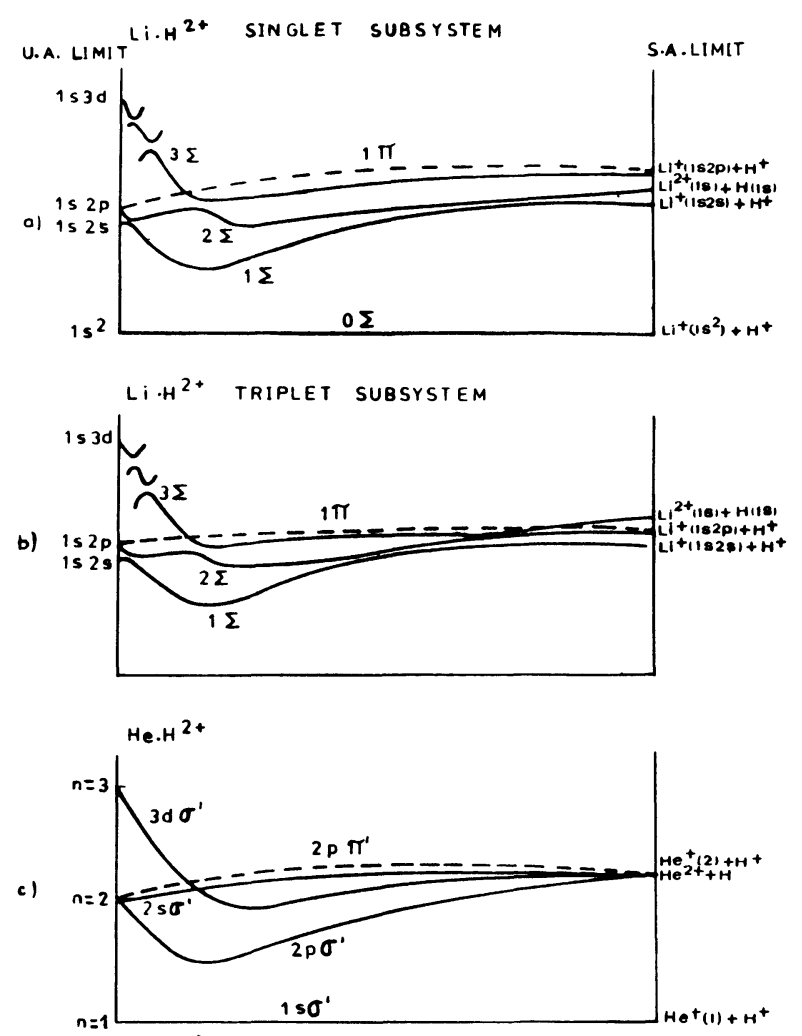

Fig. 1. - Qualitative correlation diagrams for $\mathrm{LiH}^{2+}$ : a) Singlet subsystem; b) Triplet subsystem; c) Qualitative correlation diagram for $\mathrm{HeH}^{2+}$.

\section{Theory.}

2.1 Definition OF THE OEDM AND GTO-CI METHODS. - The application of Quantum Chemical techniques to the molecular approach to atomic collisions is well known (see e.g. recent reports in [10] and [4]). Likewise, the characteristics of the OEDM method have been exposed in [1] and [2] and will not be repeated here in detail. Basically in the latter method, the wave function that represents the colliding system is expanded onto the basis functions $\psi_{j}$ of the form (the spin functions are factored out) :

$$
\psi_{j}\left(\mathbf{r}_{1}, \mathbf{r}_{2} ; R\right)=N_{j} \Theta\left[\phi_{1 j}\left(\mathbf{r}_{1}\right) \phi_{2 j}\left(\mathbf{r}_{2}\right)\right]
$$

where $N_{j}$ is a normalization factor and $\Theta$ the symmetrizer (for singlet states) or the antisymmetrizer (for triplets) operator. The OEDM orbitals $\phi_{k}$ of equation (2) are exact solutions of a one-electron Schrödinger Hamiltonian :

$$
H_{0}=-\frac{1}{2} \nabla^{2}-\frac{\xi_{\mathrm{A}}}{r_{\mathrm{A}}}-\frac{\xi_{\mathrm{B}}}{r_{\mathrm{B}}}
$$

where $r_{\mathrm{A}, \mathrm{B}}$ stands for the distance of the electron from nucleus A, B and $\xi_{\mathrm{A}}, \xi_{\mathrm{B}}$ are effective - or screened nuclear charges. Various one-electron Hamiltonians $H_{0}$ may be used to define the set of OEDM orbitals $[11,12]$. It is worth noting that, in the OEDM expan- 
sion, the collision problem can be solved by expanding directly the wave function describing the electronic state evolution onto $\left\{\psi_{j}\right\}$ :

$$
\begin{aligned}
\Psi\left(\mathbf{r}_{1}, \mathbf{r}_{2}, t\right)=\sum_{j} c_{j}(t) \psi_{j}\left(\mathbf{r}_{1}, \mathbf{r}_{2} ; R\right) \times \\
\times \exp \left[-i \int_{0}^{t}\left(S^{-1} \cdot H_{\mathrm{el}}\right)_{j j} \mathrm{~d} t^{\prime}\right]
\end{aligned}
$$

where $S$ and $H_{\mathrm{el}}$ are the overlap and Hamiltonian matrices. As the $\left\{\psi_{j}\right\}$ set is made up of non-orthogonal basis functions which are not eigenstates of $H_{\mathrm{el}}$, transitions occur through electronic, radial and rotational couplings between the basis states $\psi_{j}$. On the other hand, in the usual molecular approach, one uses a basis $\left\{\chi_{n}\right\}$ of (usually approximate) eigenfunctions of $H_{\mathrm{el}}$ of energy $E_{n}$. In the $\left\{\chi_{n}\right\}$ representation, transitions take place through mechanisms involving only radial and rotational couplings. Since electrostatic interactions are fully taken into account in this representation, the number of molecular channels that are closely coupled is usually expected to be smaller than in the OEDM approach (apparent exceptions to this rule are found, e.g., when infinite series of avoided crossings are present, but then the conventional CI approach must be abandoned and diabatic wave functions employed [14]). The price to pay for that is the irregular behaviour of the couplings as a function of internuclear distance (the more often in relation with pseudo-crossings of potential curves). Of course, one could think of expanding directly the collision wave function onto the configurations built from the GTO as done with the OEDM orbitals in (1) and (3). This would also yield smooth coupling matrix elements. However the number of terms in the expansion would be extremely large by comparison with the $\left\{\chi_{n}\right\}$ expansion of (1). In the $\mathrm{LiH}^{2+}$ case, we use more than 100 configurations (a small number for a quantum chemistry calculation) whereas from functions $\chi_{n}$ will be kept for the collision problem. One of our objectives is to show that the OEDM are, in a number of problems, a good compromise between these two desirable constraints : keep the basis in (3) small with matrix elements as smooth as possible as a function of internuclear distance. The radial couplings are smoother and generally smaller in the $\left\{\psi_{j}\right\}$ basis than in the $\left\{\chi_{n}\right\}$ basis because of both the separability of the effective Hamiltonian (2) in confocal elliptic coordinates and the constant coefficients in the linear combinations (1). Then the wave functions $\psi_{j}$ have a "character" that varies more smoothly with the internuclear distance than that of the adiabatic wave functions $\chi_{n}$. For instance, the diagonal matrix elements $\left(H_{\mathrm{el}}\right)_{i i}$ usually present less (or almost the same) number of avoided crossings than the molecular energies $E_{n}$.

An important advantage of the OEDM expansion is that Stark mixing (whose representation requires a careful selection of the atomic basis used to approximate the adiabatic wave functions) is automatically taken into account because the asymptotic form of the OEDM orbitals corresponds precisely to the Stark components. A similar comment applies to excited adiabatic states involving orbitals with a large number of modes [3].

2.2 Correlation diagram. - When one uses the adiabatic expansion in the molecular model of atomic collisions, the first step is to draw and study the corresponding qualitative energy correlation diagram. Inspection of this diagram usually permits a selection of the (finite) molecular basis $\left\{\chi_{n}\right\}$ to be employed in the calculation of the transition probabilities. In the OEDM expansion, however, the signification of a correlation diagram based on the diagonal matrix elements of $H_{\mathrm{el}}$ is less straightforward because of overlap effects and because of the existence of nondiagonal matrix elements of $H_{\mathrm{el}}$ which can be relatively large and do not necessarily vanish as the internuclear distance goes to infinity. The selection of the basis $\left\{\phi_{j}\right\}$ has then been based [2], [3] on the Barat-Lichten [15] correlation diagram. Obviously the solution of the secular equation for $H_{\mathrm{el}}$ will bring further information on the adequacy of the basis set, particularly when CI calculations are available. However the latter step is not necessarily meaningful in particular for the description of states imbedded in the continuum [3].

The adiabatic energy correlation diagram for the $\mathrm{LiH}^{2+}$ quasimolecule is drawn schematically in figure 1 for the singlet and triplet subsystems, respectively. In a following article we shall treat the reactions :

$$
\begin{aligned}
\mathrm{Li}^{2+}(1 \mathrm{~s})+\mathrm{H}(1 \mathrm{~s}) \rightarrow & \mathrm{Li}^{+}\left({ }^{1,3} \mathrm{~S} 1 \mathrm{~s} 2 \mathrm{~s}\right)+\mathrm{H}^{+} \\
& \mathrm{Li}^{+}\left({ }^{1,3} \mathrm{P} 1 \mathrm{~s} 2 \mathrm{p}\right)+\mathrm{H}^{+}
\end{aligned}
$$

The entrance channel for reactions (4) corresponds to a statistical mixture of the $2^{1} \Sigma$ and $3^{3} \Sigma$ states. For $R \rightarrow \infty$, the system $\mathrm{Li}^{2+}(1 \mathrm{~s})+\mathrm{H}(1 \mathrm{~s})$ is quasidegenerate with $\mathrm{Li}^{+}\left({ }^{1,3} \mathrm{~S} 1 \mathrm{~s} 2 \mathrm{~s}\right)$ and $\mathrm{Li}^{+}\left({ }^{1,3} \mathrm{P} 1 \mathrm{~s} 2 \mathrm{p}\right)$ (they would be degenerate if the inner $1 \mathrm{~s}$ orbital would completely screen the nucleus). The ordering of the asymptotic quasimolecular states in figure 1 is then due to the (small) $1 \mathrm{~s}-2 \mathrm{~s}$ and $1 \mathrm{~s}-2 \mathrm{p}$ orbital interactions which are different for the singlet and triplet systems.

For the singlet subsystem, the energy of $\mathrm{Li}^{2+}(1 \mathrm{~s})+$ $\mathrm{H}(1 \mathrm{~s})$ lies between those of $\mathrm{Li}^{+}\left({ }^{1} \mathrm{~S} 1 \mathrm{~s} 2 \mathrm{~s}\right)$ and $\mathrm{Li}^{+}\left({ }^{1} \mathrm{P} 1 \mathrm{~s} 2 \mathrm{p}\right)$. Consequently (see Fig. 1a), the $2{ }^{1} \Sigma$ energy has a sharp avoided crossing at $R \sim 20$ a.u. with the $1^{1} \Sigma$ state, that tends to $\mathrm{Li}^{+}\left({ }^{1} \mathrm{~S} 1 \mathrm{~s} 2 \mathrm{~s}\right)+\mathrm{H}^{+}$ as $R \rightarrow \infty$.

For the triplet subsystem, the energy of $\mathrm{Li}^{2+}(1 \mathrm{~s})+\mathrm{H}(1 \mathrm{~s})$ lies above those of $\mathrm{Li}^{+}\left({ }^{3} \mathrm{~S} 1 \mathrm{~s} 2 \mathrm{~s}\right)$ and $\mathrm{Li}^{+}\left({ }^{3} \mathrm{P} 1 \mathrm{~s} 2 \mathrm{p}\right)$. Consequently (see Fig. $1 \mathrm{~b}$ ), the $3^{3} \Sigma$ state has an energy that presents a very sharp avoided crossing at $R \sim 33$ a.u. with the $2^{3} \Sigma$ energy, and the latter energy pseudo-crosses at $R \sim 9$ a.u. that of the $1^{3} \Sigma$ state. Each of the avoided crossings mentioned corresponds to a complete interchange of 
character between the wave functions. For the energy range considered in the treatment of reactions (4) the very sharp pseudo-crossings at $R \sim 20$ and 33 a.u. are traversed diabatically and are, therefore, of little practical interest; on the contrary, partial transitions take place at the pseudo-crossing occurring for the triplet subsystem for $R \simeq 9$ a.u., which is of utmost importance in the dynamics of (4). In this paper we shall, therefore, concentrate our attention on the region of internuclear distances $R<15$ a.u. for the discussion of the couplings.

We have just seen that in the asymptotic region $R \rightarrow \infty$, the states of interest of the $\mathrm{LiH}^{2+}$ quasimolecule can be described, to a reasonable approximation, by an independent particle model in which one electron occupies the 1s orbital of $\mathrm{Li}^{2+}(1 \mathrm{~s})$ while the other electron occupies an orbital of either the hydrogen atom or the screened $\mathrm{Li}^{2+}-$ i.e. $\mathrm{He}^{+}-$ion. A comparison of the correlation diagrams of $\mathrm{LiH}^{2+}$ (Figs. 1a, b) with that of $\mathrm{HeH}^{2+}$ (Fig. 1c) indicates that this description is still a reasonable one for finite $R$. As in reference [8] the states of the $\mathrm{LiH}^{2+}$ quasimolecule can be described by assuming that one electron occupies an inner $1 \mathrm{~s} \sigma$ orbital of $\mathrm{LiH}^{3+}$, while the state of the $3 \mathrm{~d} \pi^{\prime}$ other electron is represented by a $2 \mathrm{p} \sigma^{\prime}, 2 \mathrm{~s} \sigma^{\prime}, 3 \mathrm{~d} \sigma^{\prime}, 2 \mathrm{p} \pi^{\prime}$ or $3 \mathrm{~d} \pi^{\prime}$ orbital of $\mathrm{HeH}^{2+}$. Interaction between "inner " and " outer " shell orbitals causes the energy orderings of Figs. 1a, b. Also, the crossing between the $2 \mathrm{~s} \sigma^{\prime}$ and $3 \mathrm{~d} \sigma^{\prime}$ orbital energies of $\mathrm{HeH}^{2+}$ appears as an avoided crossing between the energies of the $2^{1,3} \Sigma$ and $3^{1,3} \Sigma$ states, because of the non-separability of the full electronic Hamiltonian $H_{\mathrm{el}}$ in parabolic coordinates $\left({ }^{1}\right)$.

The previous analysis of a molecular correlation diagram is based on the early work of Hund and Mulliken (for a review, see [9]) and appears in applications of the molecular model to atomic collisions by Lichten $([13,14])$.

\section{Energies.}

According to the reasoning of the previous section, we should set up an OEDM model with two effective Hamiltonians $H_{0}$ of (2): one with $\xi_{\mathrm{A}, \mathrm{B}}=Z_{\mathrm{A}, \mathrm{B}}$, the bare nuclear charges, defines the « inner » 1s OEDM orbital; the other Hamiltonian with $\xi_{\mathrm{A}}=2, \xi_{\mathrm{B}}=1$, the nuclear charges of $\mathrm{HeH}^{2+}$, define the " outer " $1 \mathrm{~s} \sigma^{\prime}, 2 \mathrm{p} \sigma^{\prime}, 2 \mathrm{~s} \sigma^{\prime}, 3 \mathrm{~d} \sigma^{\prime}, 2 \mathrm{p} \pi^{\prime}, 3 \mathrm{~d} \pi^{\prime}$ OEDM orbitals. Inserting these OEDM orbitals in (1), with $\phi_{j_{1}}$ the " inner " and $\phi_{j_{2}}$ an " outer " orbital, we obtain a representation $\left\{\psi_{j}\right\}$. Diagonalization of $H_{\mathrm{el}}$ in the representation $\left\{\psi_{j}\right\}$ yields approximate molecular energies $E_{n}$, which are compared in table II with the results of a full $\mathrm{CI}$ calculation using the atomic basis set of table I, which has been approximately optimized

( ${ }^{1}$ ) We omit a discussion on the avoided crossings occurring at very small $R$ that are of minor importance in the dynamics of reaction (4). for the range of internuclear distances involved. The excellent agreement between both sets of energy data, calculated in such different ways, confirms the correctness of the analysis presented in the previous section, and of our choice of OEDM basis to describe the collisions (4).

The comparison between molecular energies calculated in an OEDM basis and in a CI treatment also allows us to draw some conclusions on the OEDM method. Suppose that, instead of two effective Hamiltonians $H_{0}$, we employ a simple unscreened $H_{0}$, with $\xi_{\mathrm{A}, \mathrm{B}}=Z_{\mathrm{A}, \mathrm{B}}$ in equation (2), to define all OEDM orbitals. Then the corresponding set of wave functions $\left\{\psi_{j}\right\}$ of equation (1) has the desirable property of being an orthonormal set. On the other hand, its inferiority, in the description of the static properties of the $\mathrm{LiH}^{2+}$ quasimolecule, can be easily shown. We present in table II the results of diagonalizing $H_{\mathrm{el}}$ in this new basis set $\left\{\psi_{j}\right\}$ with $\phi_{1 j}=1 \mathrm{~s} \sigma$, $\phi_{2 j}=1 \mathrm{~s} \sigma, 2 \mathrm{p} \sigma, 3 \mathrm{~d} \sigma, 4 \mathrm{f} \sigma, 2 \mathrm{p} \pi, 3 \mathrm{~d} \pi$; the corresponding molecular energies are clearly poorer than those for the screened OEDM basis. We conclude that screening permits the description of the $\mathrm{LiH}^{2+}$ quasimolecule - hence the treatment of the collision process (4) with a small number of basis wave functions $\left\{\psi_{j}\right\}$ in equation (3). This fact more than compensates for the difficulties caused by the non-orthogonality of these basis functions. Table II also shows that a more sophisticated optimization of the effective charges $\xi_{\mathrm{A}, \mathrm{B}}$ of the one electron Hamiltonians $H_{0}$ of (2) is unnecessary since the results are already better than those of our GTO-CI calculation.

Finally, it may be remarked that, in order to obtain accurate values for the molecular energies, one has to introduce in the basis $\left\{\psi_{j}\right\}$ an "extra " 1s $\sigma 1 \mathrm{~s} \sigma^{\prime}$ configuration, for both the singlet and triplet subsystem [2]. Omission of this configuration yields results which are too low for the singlet states since, then, all the approximate eigenfunctions obtained by the diagonalization of $H_{\mathrm{el}}$ contain a contribution of the exact $0^{1} \Sigma$ wave function. For triplet states, the results obtained when the $1 \mathrm{~s} \sigma 1 \mathrm{~s} \sigma^{\prime}$ configuration is omitted are too high - because in this case the contribution corresponds to a mixture of excited states, which is eliminated when $1 \mathrm{~s} \sigma 1 \mathrm{~s} \sigma^{\prime}$ is included in the basis.

\section{Couplings.}

To avoid complicating unnecessarily this article by presenting a large amount of data, we shall simply state from the start that the dynamical couplings obtained from solving the secular equation in the representation of the screened OEDM and GTO-CI basis are practically identical. This re-inforces our previous conclusion on the correctness of our choice of OEDM orbitals. Furthermore, it allows us to bet on insight into the physical origin of each coupling. Such an analysis of dynamical couplings is very 
Table I. - Exponents of the atomic Gaussian basis set employed in the $\mathrm{CI}$ calculation of the adiabatic wave functions $\left\{\chi_{j}\right\}$.

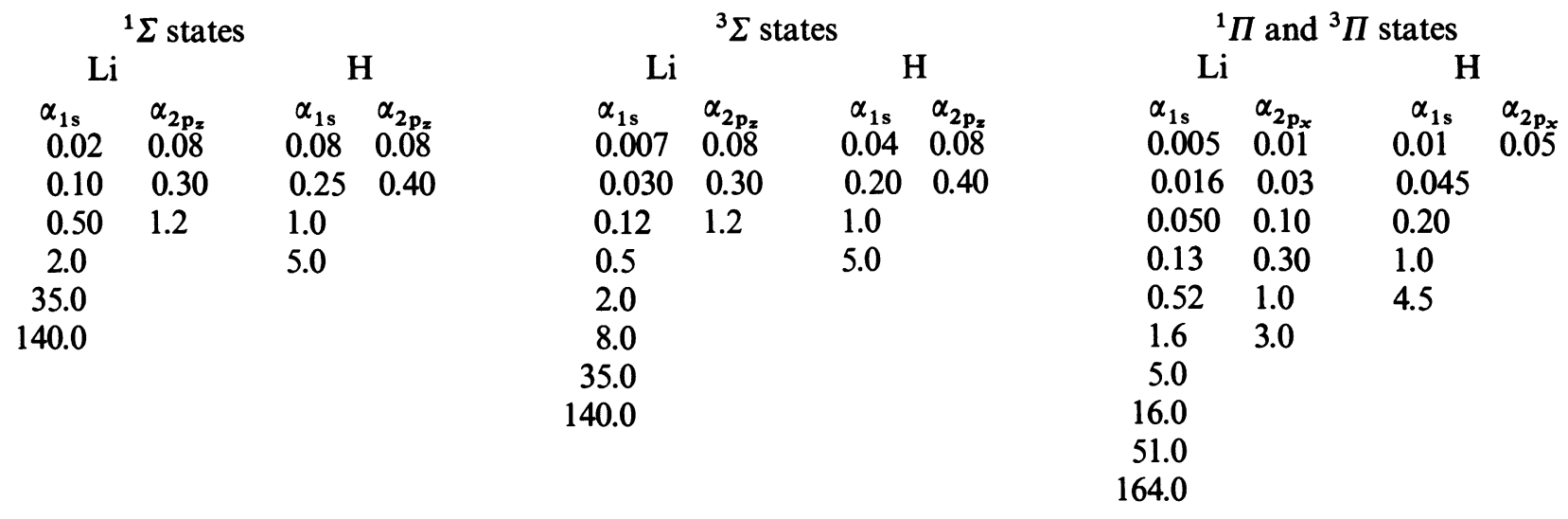

Table II. - Electronic molecular energies (in a.u.) of the $\mathrm{LiH}^{2+}$ states as a function of internuclear distance $R$ (in a.u.). A) GTO-CI calculation; B) Screened OEDM-CI calculation; C) Unscreened OEDM-CI calculation.

a) Singlet states

\begin{tabular}{rccc} 
& \multicolumn{3}{c}{${ }^{1} \Sigma$} \\
$\boldsymbol{R}$ & $\mathrm{A}$ & $\mathrm{B}$ & $\mathrm{C}$ \\
1 & 6.85393 & 6.85754 & 6.833 \\
2 & 6.35266 & 6.35963 & 6.343 \\
3 & 6.00911 & 6.01754 & 6.010 \\
4 & 5.77321 & 5.78345 & 5.779 \\
5 & 5.61312 & 5.62371 & 5.621 \\
6 & 5.50377 & 5.51305 & \\
7 & 5.42669 & 5.43502 & \\
8 & 5.37029 & 5.37820 & \\
9 & 5.32737 & 5.33507 & \\
10 & 5.29341 & 5.30104 & \\
11 & 5.26588 & 5.27340 & \\
12 & 5.24295 & 5.25046 &
\end{tabular}

\begin{tabular}{ccc}
\multicolumn{3}{c}{$2{ }^{1} \Sigma$} \\
A & B & C \\
6.44361 & 6.44901 & 6.36 \\
5.80150 & 5.80623 & 5.707 \\
5.57287 & 5.57502 & 5.481 \\
5.47295 & 5.47564 & 5.425 \\
5.42053 & 5.42589 & 5.392 \\
5.37143 & 5.37678 & \\
5.32638 & 5.33087 & \\
5.28818 & 5.29170 & \\
5.25735 & 5.26010 & \\
5.23285 & 5.23496 & \\
5.21312 & 5.21493 & \\
5.19695 & 5.19854 &
\end{tabular}

\begin{tabular}{ccc}
\multicolumn{3}{c}{${ }^{1} \Sigma$} \\
A & B & C \\
6.06225 & 6.03419 & 5.955 \\
5.55542 & 5.57033 & 5.453 \\
5.47895 & 5.48845 & 5.398 \\
5.42123 & 5.43096 & 5.327 \\
5.35070 & 5.35944 & 5.25 \\
5.29813 & 5.30607 & \\
5.25825 & 5.26576 & \\
5.22684 & 5.23419 & \\
5.20135 & 5.20871 & \\
5.18035 & 5.18771 & \\
5.16286 & 5.17029 & \\
5.14815 & 5.15560 &
\end{tabular}

\begin{tabular}{lcl}
\multicolumn{3}{c}{$1{ }^{1} \Pi$} \\
A & \multicolumn{1}{c}{ B } & \multicolumn{1}{c}{ C } \\
6.50898 & 6.52654 & 6.4752 \\
5.87535 & 5.88707 & 5.806 \\
5.61397 & 5.62650 & 5.556 \\
5.46820 & 5.47832 & 5.41 \\
5.37659 & 5.38377 & 5.314 \\
5.31394 & 5.31921 & \\
5.26856 & 5.27270 & \\
5.2412 & 5.23768 & 5.164 \\
5.20722 & 5.21037 & \\
5.18555 & 5.18847 & 5.114 \\
5.16178 & 5.17052 & \\
5.15294 & 5.15554 &
\end{tabular}

b) Triplet states

\begin{tabular}{|c|c|c|c|c|c|c|c|c|c|c|c|c|}
\hline \multirow[b]{2}{*}{$R$} & \multicolumn{3}{|c|}{$1^{3} \Sigma$} & \multicolumn{3}{|c|}{$2^{3} \Sigma$} & \multicolumn{3}{|c|}{$3^{3} \Sigma$} & \multicolumn{3}{|c|}{$1^{3} \Pi$} \\
\hline & A & B & $\mathrm{C}$ & A & B & $\mathrm{C}$ & A & B & $\mathrm{C}$ & A & B & $\mathrm{C}$ \\
\hline 1 & 7.02934 & 7.03642 & 7.022 & 6.51701 & 6.52606 & 6.4943 & 6.08967 & 6.03867 & 5.9815 & 6.57897 & 6.59113 & 6.55 \\
\hline 2 & 6.41165 & 6.42327 & 6.4125 & 5.86971 & 5.86948 & 5.8300 & 5.58765 & 5.59559 & 5.54 & 5.92337 & 5.92908 & 5.89 \\
\hline 3 & 6.03035 & 6.04186 & 6.035 & 5.64401 & 5.63949 & 5.61 & 5.52259 & 5.52883 & 5.486 & 5.65423 & 5.66040 & 5.622 \\
\hline 4 & 5.78548 & 5.79593 & 5.7917 & 5.54437 & 5.54067 & 5.521 & 5.46088 & 5.46775 & 5.425 & 5.50608 & 5.50993 & 5.470 \\
\hline 5 & 5.62093 & 5.63104 & 5.628 & 5.48436 & 5.48220 & 5.468 & 5.39304 & 5.39950 & 5.356 & 5.41350 & 5.41489 & 5.374 \\
\hline 6 & 5.50806 & 5.51729 & & 5.43346 & 5.43072 & & 5.33992 & 5.34459 & & 5.35047 & 5.35026 & \\
\hline 7 & 5.42875 & 5.43720 & & 5.39011 & 5.38618 & & 5.29851 & 5.30392 & & 5.30482 & 5.30372 & \\
\hline 8 & 5.37132 & 5.37922 & 5.3783 & 5.35445 & 5.34963 & 5.3354 & 5.26525 & 5.27037 & 5.227 & 5.27028 & 5.26867 & 5.227 \\
\hline 9 & 5.32967 & 5.33555 & & 5.32398 & 5.32041 & & 5.23827 & 5.24322 & & 5.24328 & 5.24133 & \\
\hline 10 & 5.30425 & 5.30147 & 5.301 & 5.29215 & 5.29730 & 5.2808 & 5.21628 & 5.22119 & 5.178 & 5.22150 & 5.21942 & 5.18 \\
\hline 11 & 5.28524 & 5.27839 & & 5.26467 & 5.27333 & & 5.19807 & 5.20283 & & 5.20369 & 5.20146 & \\
\hline 12 & 5.26957 & 5.26266 & & 5.24182 & 5.25046 & & 5.18288 & 5.18760 & & 5.18875 & 5.18648 & \\
\hline 15 & 5.23566 & 5.22856 & 5.211 & 5.19154 & 5.20018 & 5.20 & 5.14939 & 5.15402 & 5.111 & 5.15584 & 5.15345 & 5.11 \\
\hline
\end{tabular}

instructive per se and from the point of view of understanding the relationship between the OEDM and GTO-CI method in detail.

As stated above, we restrict our study to the physical origin of the couplings to $R<15$ a.u.
The radial couplings, which are presented in the next section, have been calculated with the methods of Harel and Salin [2] for the OEDM approach, and of Macias and Riera [16] in the GTO-CI formalism. 
The discussion below will be restricted to radial and rotational couplings, though our conclusions are not specific to these matrix elements alone. It is well known that they depend on the origin of electronic coordinates (as shown in Fig. 2 for radial couplings between ${ }^{1} \Sigma$ states) and that they do not necessarily go to zero at infinity. This conspicuous feature of the matrix elements causes difficulties in the treatment of the collision which we eliminate by the introduction of translation factors into the molecular basis set. Therefore we shall not discuss this point further.

4.1 RADIAL COUPLINGS. - The radial couplings $D_{i j}=\left\langle\psi_{i}\left|\frac{\partial}{\partial R}\right| \psi_{j}\right\rangle$ between the OEDM basis functions and between the adiabatic wave functions $D_{n m}=\left\langle\chi_{n}\left|\frac{\partial}{\partial R}\right| \chi_{m}\right\rangle$ obtained in the CI method, are presented in figures 3 and 4 respectively. To understand their physical origin and their interrelation, we first establish the formal connection between them. Solving the secular equation for $H_{\mathrm{el}}$ in the $\left\{\psi_{j}\right\}$ representation yields approximate adiabatic wave functions :

$$
\chi_{n}^{\prime} \simeq \sum_{j} \gamma_{j n} \psi_{j}
$$

where $\gamma_{n}$ is the coefficient matrix of the secular equa-

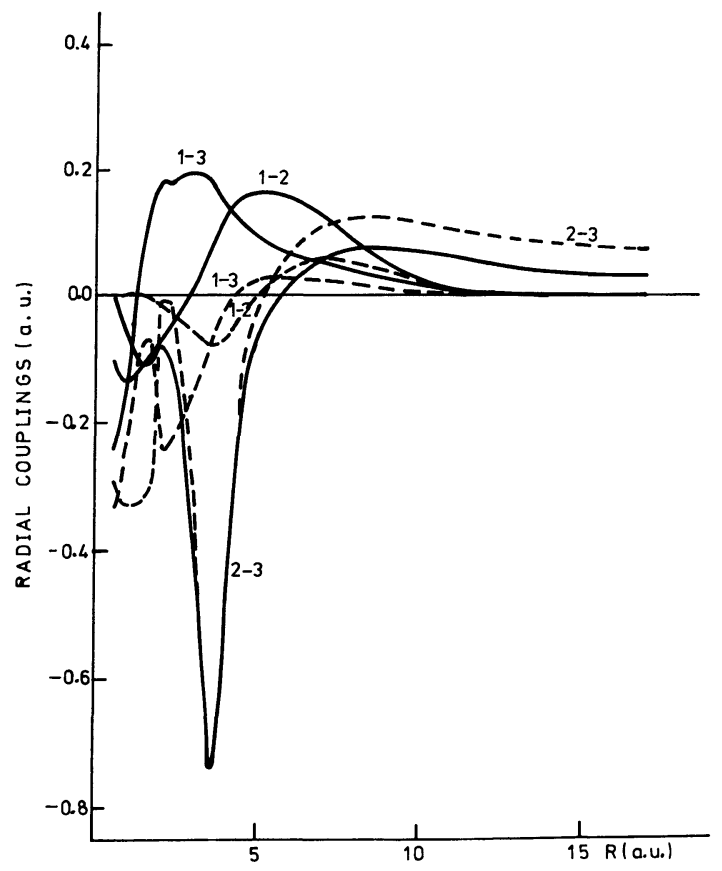

Fig. 2. - Radial couplings between the singlet adiabatic wave functions $\left\{\chi_{i}\right\}$ obtained in the CI method, for two origins of electronic coordinates :- Nuclear centre of charges ; - - - H nucleus. a) Singlet subsystem; b) Triplet subsystem. tion. Then, one can write [16] :

$$
\left\langle\chi_{n}^{\prime}\left|\frac{\partial}{\partial R}\right| \chi_{m}^{\prime}\right\rangle \simeq \gamma_{n}^{\dagger} \mathbf{S} \frac{\mathrm{d}}{\mathrm{d} R} \gamma_{m}+\gamma_{n}^{\dagger} \mathbf{D} \gamma_{m} .
$$

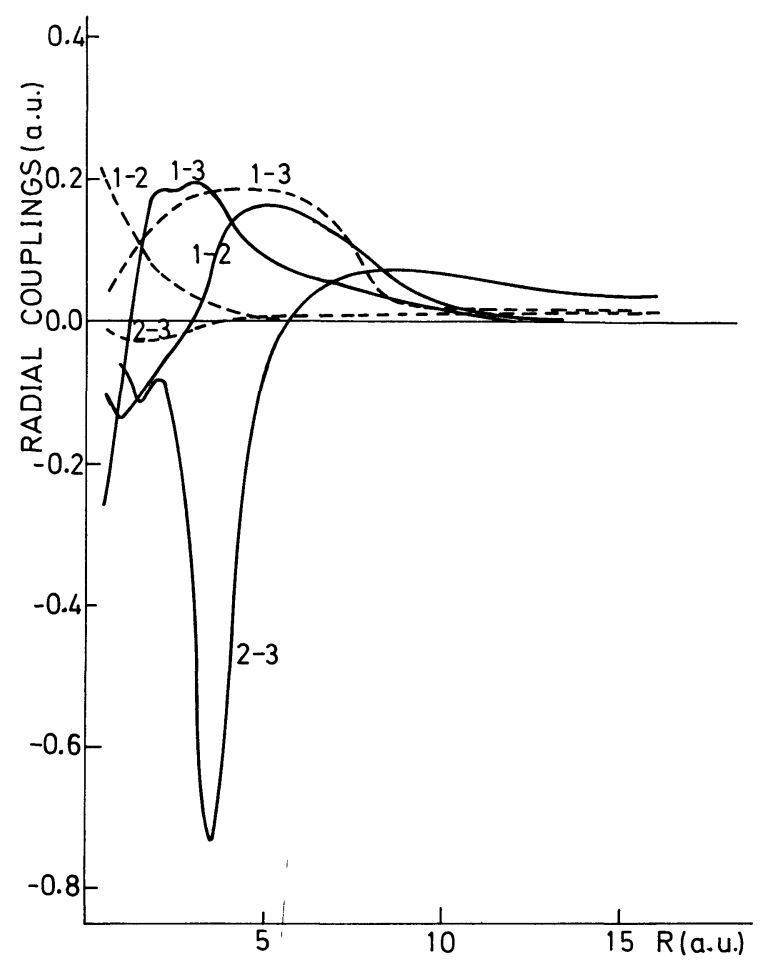

Fig. 3. - Radial couplings for the singlet subsystems : _ - - Radial coupling $\mathfrak{D}_{i j}$ between OEDM configurations; Radial coupling $D_{i j}$ between adiabatic states.

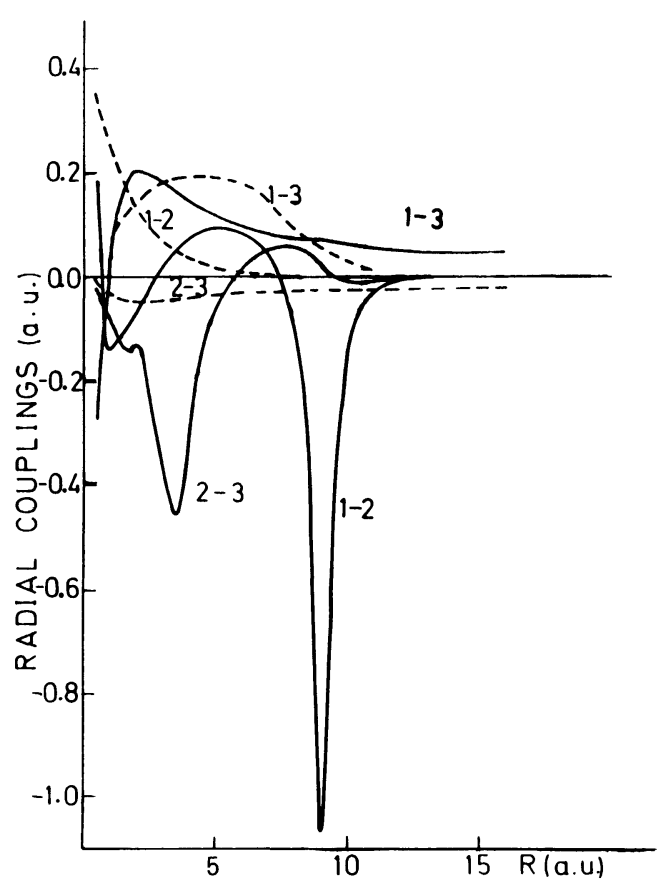

Fig. 4. - Same as figure 3 for the triplet subsystem. 
The radial coupling between the molecular wave functions is thus written as the sum of two contributions. The first term is only important when the $\left\{\psi_{j}\right\}$ basis functions interact via non-constant electrostatic or overlap effects; more explicitly, we can write this first term of (6) as [16]

$\gamma_{n}^{\dagger} \mathbf{S} \frac{\mathrm{d}}{\mathrm{d} R} \gamma_{m}=\left(E_{m}-E_{n}\right)^{-1} \gamma_{n}^{\dagger}\left(\frac{\mathrm{d}}{\mathrm{d} R} \mathbf{H}_{\mathrm{el}}-E_{m} \frac{\mathrm{d}}{\mathrm{d} R} \mathbf{S}\right) \boldsymbol{\gamma}_{m}$

where $\mathbf{S}$ and $\mathbf{H}_{\mathrm{el}}$ are the overlap and Hamiltonian matrix in the $\left\{\psi_{j}\right\}$ representation. The second term in equation (6) is due to radial couplings between the OEDM basis functions $\left\{\psi_{j}\right\}$. We shall start by studying the physical origin of the latter and see how they are mixed by electrostatic (or overlap) effects to yield the full radial coupling (6).

For the singlet and triplet case :

$$
\begin{array}{r}
\mathfrak{D}_{i j}^{1,3} \equiv\left\langle\psi_{i}\left|\frac{\partial}{\partial R}\right| \psi_{j}\right\rangle=\left\langle\phi_{2 i}\left|\frac{\partial}{\partial R}\right| \phi_{2 j}\right\rangle \mp \\
\mp\left\langle\phi_{1} \mid \phi_{2 j}\right\rangle\left\langle\phi_{1}\left|\frac{\partial}{\partial R}\right| \phi_{2 i}\right\rangle \\
+\left\langle\phi_{1} \mid \phi_{2 i}\right\rangle\left\langle\phi_{1}\left|\frac{\partial}{\partial R}\right| \phi_{2 j}\right\rangle .
\end{array}
$$

The $\mathfrak{D}_{i j}$ coupling matrix elements are thus written as the sum of a contribution due to $\mathrm{d} / \mathrm{d} R$ matrix elements between the OEDM orbitals, and a contribution due to " inner »-« outer » shell effects.

For our purposes, the most important coupling matrix elements presented in figures 3 and 4 are $D_{13}$ and $\mathfrak{D}_{23}$. The former is entirely due to the $2 \mathrm{p} \sigma^{\prime}-3 \mathrm{~d} \sigma^{\prime}$ radial coupling (see e.g. Ref. [17]). The latter is made up of two contributions : one is the (relatively small and unimportant) $2 \mathrm{~s} \sigma^{\prime}-3 \mathrm{~d} \sigma^{\prime}$ radial coupling, the other, stemming from the second and third terms of equations (8) varies little with $R$.

First consider the singlet case for large internuclear distances, the most obvious feature in figure 3 is the large value of $D_{23}^{1}$ compared to $D_{23}^{1}$. Hence the slow decrease of $D_{23}^{1}$ comes entirely from the first term in (6) and originates in the Stark effect. The $2 s \sigma^{\prime}$ and $3 \mathrm{~d} \sigma^{\prime}$ orbitals are Stark hybrids, hence the Stark coupling is absent from $\mathfrak{D}_{23}^{1}$. Instead, the basis functions $\psi_{2}\left(1 \mathrm{~s} \sigma 2 \mathrm{~s} \sigma^{\prime}\right)$ and $\psi_{3}\left(1 \mathrm{~s} \sigma 3 \mathrm{~d} \sigma^{\prime}\right)$ interact for $R<20$ a.u. via $H_{\mathrm{el}}$ and the variation of this interaction with $R$ is responsible for the Stark coupling $D_{23}^{1}$ between the adiabatic wave functions $\chi_{2}$ and $\chi_{3}$. We can confirm this by comparing $D_{23}^{1}$ with the asymptotic value evaluated in the appendix :

$$
D_{23}^{1} \sim \frac{a R}{R^{4}+a^{2}} \underset{R \rightarrow \infty}{\sim} a R^{-3} .
$$

Our values of $\mu$ and $\Delta E$ give $a=66.4$ a.u. which fits closely the results of figure 3 .
For $R<6$ a.u., the diagonal matrix elements $\left(S^{-1} H\right)_{22}$ and $\left(S^{-1} H\right)_{33}$ cross for $R \simeq 3.75$ a.u. Hence, apart from a "background " contribution $D_{23}^{1}$ varying smoothly with $R, D_{23}^{1}$ shows a peak around $R=3.75$ a.u. because the adiabatic energies do not cross. The reason why the contribution to $D_{23}^{1}$ of the Stark effect and that of the crossing between OEDM are of opposite signs has been explained in reference [5] and [6]. In short, for large $R$ the Stark effect stabilizes (yields a lower energy for) the " bonding " hybrid wave function $\left|2^{1} \Sigma\right\rangle$ which correlates smoothly for $R \rightarrow 0$ to the united atom limit 1s $3 \mathrm{~d}$, with respect to the "anti-bonding" hybrid wave function $\left|3^{1} \Sigma\right\rangle$ that correlates to $1 \mathrm{~s} 2 \mathrm{~s}$. In the avoided crossing region smooth correlation is violated [5], and for smaller internuclear distance the " bonding " hybrid corresponds to the wave function $\left|3^{1} \Sigma\right\rangle$. Since the adiabatic wave functions $\left|2^{1} \Sigma\right\rangle$ and $\left|3^{1} \Sigma\right\rangle$ change continuously from a " bonding " to an "antibonding" character and vice-versa, the corresponding $2{ }^{1} \Sigma-3{ }^{1} \Sigma$ radial coupling must reflect this undoing of the Stark hybridization and changes sign accordingly. As a result, for $R<6$ a.u., the $\left\langle 2^{1} \Sigma\left|\frac{\partial}{\partial R}\right| 3^{1} \Sigma\right\rangle$ matrix element is the difference between two contributions : a Lorentzian peak of area $\sim \pi / 2$ due to the avoided crossing between the $2^{1} \Sigma$ and $3^{1} \Sigma$ energy curves (linear model [19]) and the Stark coupling (9).

The strongest coupling between OEDM configuration is $D_{13}^{1}$ for $R>2$ a.u. and $\mathcal{D}_{12}^{1}$ below $R=2$ a.u. For $R>10$ a.u., this value of $\mathfrak{D}_{13}^{1}$ contributes to both $D_{12}^{1}$ and $D_{13}^{1}$ because of the Stark hybridation phenomenon discussed earlier. For $3.75<R<10$, however, it contributes mostly to $D_{12}^{1}$ because, in this region, the overlap of $\psi_{3}$ is much larger with $\chi_{2}$ than with $\chi_{3}$. Below $R_{c}=3.75$, the states $\chi_{2}$ and $\chi_{3}$ exchange their character. This produces the following transformation :

$$
\begin{aligned}
& D_{12}^{1}\left(R_{\mathrm{c}}+\Delta R\right) \simeq D_{13}^{1}\left(R_{\mathrm{c}}-\Delta R\right) \\
& D_{12}^{1}\left(R_{\mathrm{c}}-\Delta R\right) \simeq-D_{13}^{1}\left(R_{\mathrm{c}}+\Delta R\right) .
\end{aligned}
$$

Clearly $D_{12}^{1} \simeq-D_{12}^{1}$ for $R<2.5$ atomic units. However, both $D_{12}^{1}$ and $D_{13}^{1}$ are perturbed at small internuclear distances by pseudo-crossings that are of no importance for the collision process.

The above discussion completely explains the values of the matrix elements $D_{i j}^{1}$ in terms of the simpler $\mathfrak{D}_{i j}^{1}$ matrix elements and OEDM properties. A similar discussion can be carried out for the triplet case. The similarity between the $\mathfrak{D}_{i j}^{1}$ and $\mathfrak{D}_{i j}^{3}$ matrix elements in the singlet and triplet case is obvious from a comparison of figure 3 with figure 4 . However the $D_{i j}^{1}$ and $D_{i j}^{3}$ are quite different. It is an interesting consequence of the OEDM method that the strongly different $D_{i j}$ in the singlet and triplet case can be easily interpreted from the very similar behaviour of the OEDM properties. In addition to the pseudo- 
crossing between $\chi_{2}$ and $\chi_{3}$ around $R_{\mathrm{c}}^{1}=3.75$ a.u., there is a pseudo-crossing between $\chi_{1}$ and $\chi_{2}$ around $R_{\mathrm{c}}^{2}=9$ a.u. Therefore the Stark coupling appears in $D_{13}$ for $R>R_{\mathrm{c}}^{2}$.

We get the following correlations :

$$
\begin{aligned}
& D_{13}^{3}\left(R_{\mathrm{c}}^{2}-\Delta R\right) \simeq-D_{23}^{3}\left(R_{\mathrm{c}}^{2}+\Delta R\right) \\
& D_{23}^{3}\left(R_{\mathrm{c}}^{2}-\Delta R\right) \simeq+D_{13}^{3}\left(R_{\mathrm{c}}^{2}+\Delta R\right) .
\end{aligned}
$$

For distances smaller than $R_{\mathrm{c}}^{2}$, the triplet and singlet $D_{i j}$ are qualitatively identical.

4.2 Rotational COUPLINGS. - The rotational couplings $\mathscr{L}_{i}=\left\langle\psi_{i}\left|i L_{y}\right| \psi_{4}\right\rangle$ between the OEDM configurations and $L_{i}=\left\langle\chi_{i}\left|i L_{y}\right| \chi_{4}\right\rangle$ between the adiabatic functions are given in figures 5 and 6 . Let us first establish the relation between $L_{i}$ and $\mathcal{L}_{i}$. If we admit that $\chi_{4} \simeq \psi_{4}$ :

$$
L_{i}=\sum_{j} \gamma_{j i} \mathcal{L}_{j}
$$

Furthermore, from the form of the configurations considered in the present work :

$$
\begin{aligned}
\mathcal{L}_{i}^{1,3}=\left\langle\Phi_{i}\left|i L_{y}\right| \Phi_{2 \mathrm{p} \pi^{\prime}}\right\rangle & \pm\left\langle\phi_{1} \mid \phi_{i}\right\rangle \times \\
& \times\left\langle\phi_{1}\left|i L_{y}\right| \phi_{2 \mathrm{p} \pi^{\prime}}\right\rangle .
\end{aligned}
$$

Again the variation of $\mathcal{L}_{i}^{1,3}$ with internuclear distance is quite simple but the difference between singlet and triplet states is appreciable. This difference is due to the second term in (13) which shows the importance of overlap effects between the inner and outer orbitals in our OEDM basis.

Let us first consider the singlet case (Fig. 5). For $R \rightarrow 0, L_{1}^{1}$ and $\mathcal{L}_{1}^{1}$ tend to one because they both tend to a matrix element between a $2 p_{0}$ and $2 p_{x}$ state. Similarly both $L_{1}^{1}$ and $\mathcal{L}_{1}^{1}$ tend exponentially to zero for $R \rightarrow \infty$.

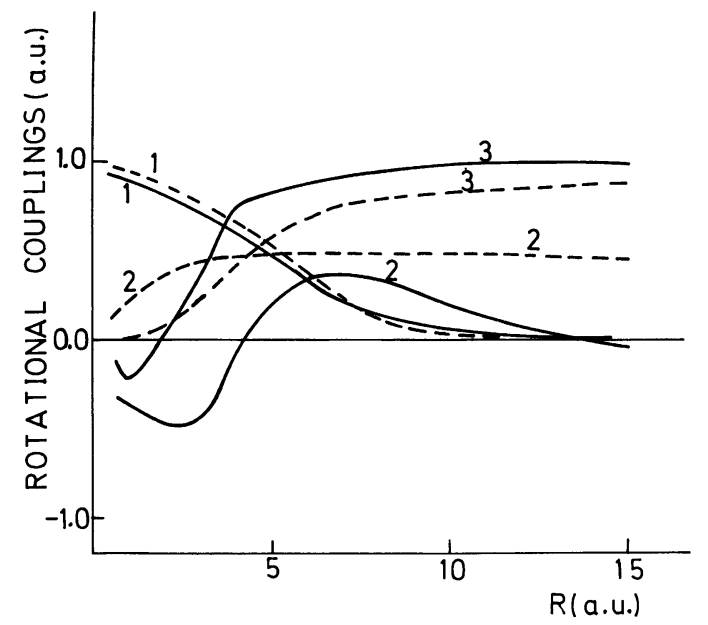

Fig. 5. - Rotational couplings for the singlet subsystem : - - - Rotational coupling $\mathcal{L}_{i j}$ between OEDM configurations; - Rotational coupling $L_{i j}$ between adiabatic states.
For distances of the order of 7-10 a.u., one has $L_{2}^{1} \simeq \mathcal{L}_{2}^{1}$ and $L_{3}^{1} \simeq \mathcal{L}_{3}^{1}$. However, for large distances, as the OEDM orbitals correspond to Stark hybrides $L_{2}^{1} \rightarrow 1 / \sqrt{2}\left(\mathcal{L}_{2}^{1}-\mathcal{L}_{3}^{1}\right)$ and $L_{3} \rightarrow 1 / \sqrt{2}\left(\mathcal{L}_{2}^{1}+\mathcal{L}_{3}^{1}\right)$.

Around $R_{\mathrm{c}}=3.75$ a.u., a complete change of character from $\chi_{2}$ to $\chi_{3}$ and vice-versa would give the same correlation rules on $L_{2}^{1}$ and $L_{3}^{1}$ between $R>R_{\mathrm{c}}$ and $R<R_{\mathrm{c}}$ as for the radial couplings namely $L_{2}^{1}\left(R_{\mathrm{c}}-\Delta R\right)=-L_{3}^{1}\left(R_{\mathrm{c}}+\Delta R\right)$ and $L_{3}^{1}\left(R_{\mathrm{c}}-\Delta R\right)$ $=+L_{2}^{1}\left(R_{\mathrm{c}}+\Delta R\right)$. This is less obvious, however, in figure 5 since the $\chi$ can no longer be expressed as a good approximation by a single $\psi$.

A similar interpretation can be done for the triplet case (Fig. 6). For $R \gg R_{\mathrm{c}}^{2}=9$ a.u., we get :

$$
L_{1}^{3} \rightarrow \frac{1}{\sqrt{2}}\left(\mathcal{L}_{3}^{3}-\mathcal{L}_{2}^{3}\right), \quad L_{3}^{3} \rightarrow \frac{1}{\sqrt{2}}\left(\mathcal{L}_{3}^{3}+\mathcal{L}_{2}^{3}\right)
$$

and $L_{2}^{3}$ decreases exponentially. Around $R_{\mathrm{c}}^{2}=9$ a.u., the states $\chi_{1}$ and $\chi_{2}$ exchange their characters so that, for $R<R_{\mathrm{c}}^{2}, L_{1}^{3} \simeq \mathfrak{L}_{1}^{3}$. The variation of $L_{2}^{3}$ is less simple because this region is also that where the adiabatic $\chi$ states evolve toward Stark states as $R$ decreases - see above. The situation around $R_{\mathrm{c}}^{1}=3.75$ a.u. is similar to that of the singlet case.

\section{Conclusions.}

In the present article we have performed a detailed analysis of the properties of the wave functions $\left\{\psi_{j}\right\}$ and $\left\{\chi_{n}\right\}$ in the OEDM and CI representations of the $\mathrm{LiH}^{2+}$ quasimolecule, and we have established the close relationship between the coupling matrix elements in both representations. From our analysis, the correctness of the assignation of molecular orbital symbols in correlation with energy diagrams (see examples [4] and [18]) describes the molecular states which becomes apparent, as shown by an explicit comparison between energies and couplings.

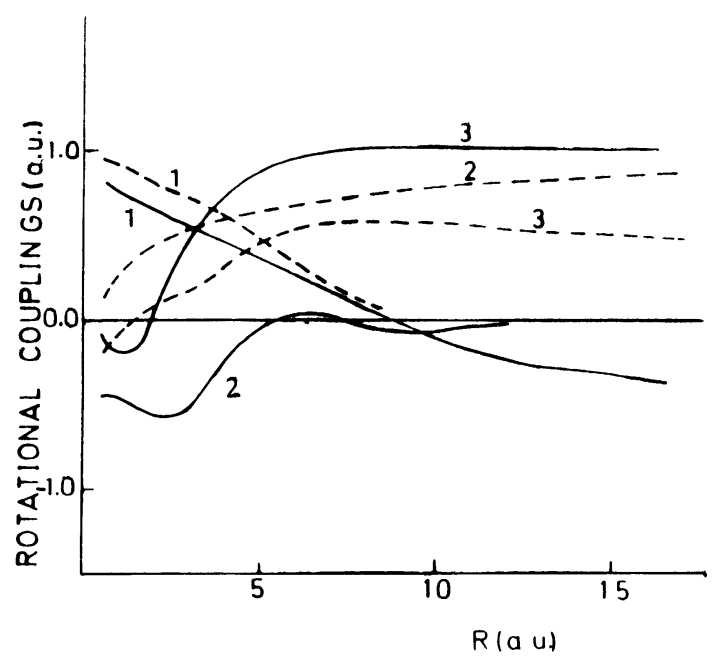

Fig. 6. - Same as figure 5 for the triplet subsystem. 
We stress the fact that intuitive " one-active electron » pictures, when only one molecular orbital differs for all molecular states involved in a given process, as in the present case, do not mean that one can neglect the interaction between the " outer $"$ orbital describing this electron, and the " inner " orbital describing the " passive " electron. In the present example, it is precisely this "inner-outer » orbital interaction that is responsible for the energy ordering of figure 4, and for couplings which will be shown in the following paper to be very important in the description of the dynamics of reactions (4) : the peak in

$$
\left\langle 1^{3} \Sigma\left|\frac{\partial}{\partial R}\right| 2^{3} \Sigma\right\rangle
$$

(see Fig. 4) and the part of matrix elements

$$
\left\langle 2^{1,3} \Sigma\left|\frac{\partial}{\partial R}\right| 3^{1,3} \Sigma\right\rangle
$$

which is not cancelled out when translation factors are introduced into the basis. Even in the OEDM picture it should be remarked that "inner-outer " interaction gives rise to a non-negligible contribution to the dynamical couplings (see e.g. Eqs. (8) and (13)).

\section{Appendix.}

To explain the form of the radial coupling due to Stark mixing it is useful to set up a simple model which only takes into account the formation of Stark hybrids from a set of quasi-degenerate molecular states. We shall restrict ourselves here to a two-state model which is directly relevant to the cases presented in this paper :

$$
\mathbf{H}=\left(\begin{array}{lrr}
E_{1}(\infty) & -\frac{Z Z^{\prime}}{R} & -\frac{Z \mu}{R^{2}} \\
-\frac{Z \mu}{R^{2}} & E_{2}(\infty)-\frac{Z Z^{\prime}}{R}
\end{array}\right)
$$

where $Z$ is the charge of the approaching ion that Stark couples two states of an ion of charge $Z^{\prime}$; $\mu$ is the transition dipole moment between these two states. Diagonalization of (A.1) :

$$
(\mathbf{H}-E \mathbf{I}) \mathbf{c}=\mathbf{0}, \quad \mathbf{c}=\left(\begin{array}{rr}
\cos \theta & \sin \theta \\
-\sin \theta & \cos \theta
\end{array}\right)
$$

yields the adiabatic energies $E_{1}$ and $E_{2}$.

From the condition :

$$
0=\mathbf{c}_{1}^{\dagger} \mathbf{H c}_{2}=-\frac{1}{2} \Delta E \sin 2 \theta-\frac{Z \mu}{R^{2}} \cos 2 \theta
$$

with $\Delta E=E_{2}(\infty)-E_{1}(\infty)$, one has :

$$
\theta=-\frac{1}{2} \tan ^{-1}\left(\frac{2 Z \mu}{\Delta E R^{2}}\right)=-\frac{1}{2} \tan ^{-1}\left(\frac{a}{R^{2}}\right)
$$

with $a=\frac{2 Z \mu}{\Delta E}$. Then, the Stark mixing gives rise to a radial coupling between the adiabatic wave functions :

$$
\mathrm{c}_{1}^{\dagger} \frac{\mathrm{d}}{\mathrm{d} R} \mathrm{c}_{2}=\frac{\mathrm{d} \theta}{\mathrm{d} R}=\frac{a R}{R^{4}+a^{2}} .
$$

We notice that the area below this coupling is $\int_{0}^{\infty} \mathrm{c}_{1}^{\dagger} \frac{\mathrm{d}}{\mathrm{d} R} \mathrm{c}_{2} \mathrm{~d} R=\frac{\pi}{4}$, independently of the value of $\Delta E$ and $\mu$.
[1] Harel, C. and Salin, A., J. Phys. B 13 (1980) 785.

[2] Harel, C. and Salin, A., J. Phys. B 15 (1983) 55.

[3] Bliman, S., Hitz, D., JacQuot, B., Harel, C. and Salin, A., J. Phys. B 16 (1983) 2849.

[4] Macias, A. and Riera, A., Phys. Rep. 90 (1982) 299.

[5] Green, T. A., Browne, J. C., Michels, H. H. and Madsen, M. M., J. Chem. Phys. 61 (1974) 5198.

[6] Green, T. A., Michels, H. H., Browne, J. C. and Madsen, M. M., J. Chem. Phys. 61 (1974) 5186.

[7] Green, T. A., Michels, H. H., Browne, J. C. and Madsen, M. M., J. Chem. Phys. 69 (1978) 101.

[8] Macias, A., Riera, A. and Yáñez, M., Phys. Rev. A 27 (1983) 206.
[9] Roothaan, C. C. J., Rev. Mod. Phys. 23 (1951) 69.

[10] Garrett, B. C. and Truhlar, D. G., Theor. Chem. Adv. Prospect. 6A (1981) 215.

[11] Aubert, M., Bessis, N. and Bessis, G., Phys. Rev. A 10 (1974) 51.

Aubert, M., Bessis, N. and Bessis, G., Phys. Rev. A 10 (1974) 61.

[12] Teller, E., Sahlin, H. L., Physical Chemistry : an Advanced Treatise (Academic Press, New York) 1970, Vol. V.

[13] Lichten, W., Phys. Rev. 131 (1963) 229.

[14] Lichten, W., Phys. Rev. A 139 (1965) 27.

Lichten, W., Phys. Rev. 164 (1967) 131. 
[15] Barat, M., Lichten, W., Phys. Rev. A 11 (1972) 211.

[16] Macias, A. and Riera, A., J. Phys. B 10 (1977) 867 ; 11 (1978) 1077.

[17] Piacentini, R. D. and Salin, A., J. Phys. B 10 (1977) 1515.

[18] Falcon, C., Macias, A., Riera, A. and Salin, A., J. Phys. B 14 (1981) 1983.
[19] Nikitin, E. E., in Chemische Elementarprozesses (Springer Verlag, Berlin) 1968, p. 43.

Macias, A., Riera, A. and YÁñez, M., Phys. Rev. A 23 (1981) 2941.

[20] Eichler, J., Wille, U., Fastrup, B. and TAulbJERG, K., Phys. Rev. A 14 (1976) 707. 